Article

\title{
On Hadamard Type Fractional Inequalities for Riemann-Liouville Integrals via a Generalized Convexity
}

\author{
Tao Yan ${ }^{1}\left(\mathbb{D}\right.$, Ghulam Farid $^{2}$ (D) Hafsa Yasmeen ${ }^{2}$ and Chahn Yong Jung ${ }^{3, *(D)}$ \\ 1 School of Computer Science, Chengdu University, Chengdu 610106, China; yantao@cdu.edu.cn \\ 2 Department of Mathematics, COMSATS University Islamabad, Attock Campus, Attock 43600, Pakistan; \\ ghlmfarid@ciit-attock.edu.pk (G.F.); hafsatariq467@gmail.com (H.Y.) \\ 3 Department of Business Administration, Gyeongsang National University, Jinju 52828, Korea \\ * Correspondence: bb5734@gnu.ac.kr
}

check for

updates

Citation: Yan, T.; Farid, G.; Yasmeen,

H.; Jung, C.Y. On Hadamard Type

Fractional Inequalities for

Riemann-Liouville Integrals via a Generalized Convexity. Fractal Fract. 2022, 6, 28. https://doi.org/ $10.3390 /$ fractalfract 6010028

Academic Editor: Carlo Cattani

Received: 9 November 2021

Accepted: 8 December 2021

Published: 3 Jaunary 2022

Publisher's Note: MDPI stays neutral with regard to jurisdictional claims in published maps and institutional affiliations.

Copyright: (c) 2022 by the authors. Licensee MDPI, Basel, Switzerland. This article is an open access article distributed under the terms and conditions of the Creative Commons Attribution (CC BY) license (https:// creativecommons.org/licenses/by/ $4.0 /)$.

\begin{abstract}
In the literature of mathematical inequalities, convex functions of different kinds are used for the extension of classical Hadamard inequality. Fractional integral versions of the Hadamard inequality are also studied extensively by applying Riemann-Liouville fractional integrals. In this article, we define $(\alpha, h-m)$-convex function with respect to a strictly monotone function that unifies several types of convexities defined in recent past. We establish fractional integral inequalities for this generalized convexity via Riemann-Liouville fractional integrals. The outcomes of this work contain compact formulas for fractional integral inequalities which generate results for different kinds of convex functions.
\end{abstract}

Keywords: Riemann-Liouville integrals; hadamard inequality; $(\alpha, h-m)$-convex function; convex function

MSC: 33E12; 26A33; 26A51

\section{Introduction}

Convexity theory has a rich history and has emerged as a powerful tool over the last century. It has played a significant role in extensions and generalizations of classical results. It is also well known that convex functions are closely related to the theory of inequalities. Therefore, due to widespread applications, many inequalities for convex functions have been applied in pure and applied mathematics including financial mathematics, analysis, optimization theory, economics and graph theory, etc.

Let $I \subseteq \mathbb{R}$ is a non-empty interval in $\mathbb{R}$. Then a real valued function $f: I \rightarrow \mathbb{R}$ is said to be convex function, if $f(a t+(1-t) b) \leq t f(a)+(1-t) f(b)$, for all $a, b \in I$ and $t \in[0,1]$.

We recall the definition of $(\alpha, h-m)$-convex function as follows:

Definition 1 ([1]). Let $J \subseteq \mathbb{R}$ be an interval containing $(0,1)$ and let $h: J \rightarrow \mathbb{R}$ be a non-negative function. We say that $f:[0, b] \rightarrow \mathbb{R}$ is $a(\alpha, h-m)$-convex function, if $f$ is non-negative and for all $x, y \in[0, b],(\alpha, m) \in[0,1]^{2}$ and $t \in(0,1)$, one has

$$
f(t x+m(1-t) y) \leq h\left(t^{\alpha}\right) f(x)+m h\left(1-t^{\alpha}\right) f(y)
$$

The popular Hadamard inequality is very nice geometric visualization of convex function and it is stated as follows:

If $f:[a, b] \rightarrow \mathbb{R}$ is a convex function on interval $[a, b] \subset \mathbb{R}$ and $x, y \in[a, b]$ where $x<y$, then the following inequality holds:

$$
f\left(\frac{x+y}{2}\right) \leq \frac{1}{y-x} \int_{x}^{y} f(\xi) d \xi \leq \frac{f(x)+f(y)}{2}
$$


For concave function on $[a, b]$, both the inequalities in (2) will be reversed. Many researchers have obtained several generalizations, extensions and variants of this inequality, see [2-20] and references therein.

In the past few decades, fractional calculus has become an emerging and active field of research. Its history is as old as classical calculus. Nowadays, it not only deals with the study of so-called fractional order integrals but it also deals with the derivative operators and their applications. A lot of mathematical modeling addresses complex real life problems, and the use of fractional calculus spans diverse fields of engineering and sciences including but not limited to circuits systems, viscoelasticity, ecological systems, fluid dynamics and signal processing. Based on their importance in fractional calculus, a comprehensive account of fractional integral and derivative operators can be found in [21-25].

In this paper, we define a new class of functions, named as $(\alpha, h-m)$-convex function with respect to a strictly monotone function. This class of functions will unify several types of convexities, also the Hadamard fractional integral inequalities are established for this new class of functions keeping Riemann-Liouville fractional integrals.

The classical Riemann-Liouville integrals of fractional order and the Hadamard inequality for these integrals are given in the following definition and theorems, respectively:

Definition 2. Let $f \in L_{1}[a, b]$. Then the left and right-sided Riemann-Liouville fractional integral operators of the function $f$ of fractional order $\mu>0$ are given as follows:

$$
I_{a^{+}}^{\mu} f(x)=\frac{1}{\Gamma(\mu)} \int_{a}^{x}(x-t)^{\mu-1} f(t) d t, \quad x>a,
$$

and

$$
I_{b^{-}}^{\mu} f(x)=\frac{1}{\Gamma(\mu)} \int_{x}^{b}(t-x)^{\mu-1} f(t) d t, \quad x<b .
$$

The fractional Hadamard inequalities for Riemann-Liouville integrals are given in the next two theorems.

Theorem 1 ([14]). Let $f \in L_{1}[a, b]$ be positive and convex function on $[a, b]$. Then the following inequality holds:

$$
f\left(\frac{a+b}{2}\right) \leq \frac{\Gamma(\mu+1)}{2(b-a)^{\mu}}\left[I_{a^{+}}^{\mu} f(b)+I_{b^{-}}^{\mu} f(a)\right] \leq \frac{f(a)+f(b)}{2},
$$

with $\mu>0$.

Theorem 2 ([15]). Let $f \in L_{1}[a, b]$ be positive and convex function on $[a, b]$. Then the following inequality holds:

$$
f\left(\frac{a+b}{2}\right) \leq \frac{2^{\mu-1} \Gamma(\mu+1)}{(b-a)^{\mu}}\left[I_{\left(\frac{a+b}{2}\right)^{+}}^{\mu} f(b)+I_{\left(\frac{a+b}{2}\right)^{-}}^{\mu} f(a)\right] \leq \frac{f(a)+f(b)}{2},
$$

with $\mu>0$.

The definition of $k$-fractional Riemann-Liouville integrals is stated as follows:

Definition 3 ([26]). Let $f \in L_{1}[a, b]$. Then the left and right sided $k$-fractional Riemann-Liouville integrals of the function $f$ of fractional order $\mu>0, k>0$ are defined as follows:

$$
{ }_{k} I_{a^{+}}^{\mu} f(x)=\frac{1}{k \Gamma_{k}(\mu)} \int_{a}^{x}(x-t)^{\frac{\mu}{k}-1} f(t) d t, \quad x>a,
$$


and

$$
{ }_{k} I_{b^{-}}^{\mu} f(x)=\frac{1}{k \Gamma_{k}(\mu)} \int_{x}^{b}(t-x)^{\frac{\mu}{k}-1} f(t) d t, \quad x<b,
$$

where $\Gamma_{k}(\mu)=\int_{0}^{\infty} t^{\mu-1} e^{-\frac{t^{k}}{k}} d t$.

Using the fact $\Gamma_{k}(\mu)=k^{\frac{\mu}{k}-1} \Gamma\left(\frac{\mu}{k}\right)$ in (3) and (4) after replacing $\mu$ by $\frac{\mu}{k}$, one can get

$$
\begin{aligned}
& k^{\frac{-\mu}{k}} I_{a^{+}}^{\frac{\mu}{k}, \psi} f(x)={ }_{k} I_{a^{+}}^{\mu, \psi} f(x), \\
& k^{\frac{-\mu}{k}} I_{b^{-}}^{\mu, \psi} f(x)={ }_{k} I_{b^{-}}^{\mu, \psi} f(x) .
\end{aligned}
$$

In the upcoming section, we define $(\alpha, h-m)$-convexity with respect to a strictly monotone function. In Section 3, Hadamard inequalities for $(\alpha, h-m)$-convexity with respect to a monotone function using integrals (3) and (4) are derived. Furthermore, we have deduced a lot of fractional versions of Hadamard inequalities published in $[1,4,7-11,14,15,27-32]$.

\section{Some New Notions of Convexity}

First, in the following we define $(\alpha, h-m)$-convex function with respect to a strictly monotone function:

Definition 4. Let $K \subseteq \mathbb{R}$ be an interval containing $(0,1)$ and let $h: K \rightarrow \mathbb{R}$ be a non-negative function. Let $I, J$ be intervals in $\mathbb{R}$ and $\psi: J \rightarrow \mathbb{R}$ be strictly monotonic function and $J \subset I$. Then $f: I \rightarrow \mathbb{R}$ is said to be $(\alpha, h-m)$-convex with respect to $\psi$, if $f \circ \psi^{-1}$ is $(\alpha, h-m)$-convex function.

Mathematically we have:

$$
f \circ \psi^{-1}(t x+m(1-t) y) \leq h\left(t^{\alpha}\right) f \circ \psi^{-1}(x)+m h\left(1-t^{\alpha}\right) f \circ \psi^{-1}(y),
$$

holds for $(\alpha, m) \in(0,1] \times[0,1]$ and $t \in(0,1)$ provided $t x+m(1-t) y \in \operatorname{Im}(\psi)$ for $x, y \in \operatorname{Im}(\psi)$. For $\psi(x)=x$ in (11), we get the definition of $(\alpha, h-m)$-convex function.

Remark 1. Let $\psi(x)=x^{p} ; p \in \mathbb{R}-\{0\}, x \in(0, \infty)$. Then $\psi^{-1}(x)=x^{\frac{1}{p}}$, one can have the following inequality

$$
f\left((t x+m(1-t) y)^{\frac{1}{p}}\right) \leq h\left(t^{\alpha}\right) f\left(x^{\frac{1}{p}}\right)+m h\left(1-t^{\alpha}\right) f\left(y^{\frac{1}{p}}\right) .
$$

By replacing $x$ with $x^{p}$ and $y$ with $y^{p}$ in (12), the definition of $(\alpha, h-m)$-p-convexity defined in [33] can be obtained. Moreover after replacing $x$ with $x^{p}, y$ with $y^{p}$ and taking $\alpha=m=1$ in (12), the definition of $(p, h)$-convex function defined in [34] can be obtained. Similarly, one can obtain the definitions of $p$-convexity by replacing $x$ with $x^{p}, y$ with $y^{p}$ and setting $\alpha=m=1, h(t)=t$ defined in [35], $(\alpha, h-m)$-convexity stated in [1] can be obtained by setting $p=1$ and if $\alpha=p=1, h(t)=t^{s}$ in (12), the (s, m)-convexity can be obtained stated in [36].

Remark 2. Let $\psi(x)=\ln x, x \in(0, \infty)$. Then $\psi^{-1}(x)=\exp x$, one can have the following inequality

$$
f(\exp (t x+m(1-t) y)) \leq h\left(t^{\alpha}\right) f(\exp x)+m h\left(1-t^{\alpha}\right) f(\exp y) .
$$

By replacing $x$ with $\ln x$ and replacing $y$ with $\ln y$, the definition of $(\alpha, h-m)$-GAconvexity is reproduced as given below:

$$
f(\exp (t \ln x+m(1-t) \ln y)) \leq h\left(t^{\alpha}\right) f(x)+m h\left(1-t^{\alpha}\right) f(y) .
$$


If $\alpha=1$ in (14), we get the definition of $(h-m)$-geometrically-arithmetically-convex function (( $h-m)$-GA-convex function) given in [37]. If $\alpha=m=1, h(t)=t^{s}$ in (14), we get the definition of $(s, m)$-geometrically-arithmetically-convex function $((s, m)$-GA-convex function) given in [37]. If $m=1$ and $h(t)=t$ in (14), we get the definition of $(\alpha, m)$ geometrically-arithmetically-convex function $((\alpha, m)$-GA-convex function) given in [19]. If $h(t)=t^{-s}$ with $\alpha=1$ in (14), the following definition of $(s, m)$-Godunova-Levin-GA function can be obtained:

$$
f(\exp (t \ln x+m(1-t) \ln y)) \leq \frac{f(x)}{t^{s}}+\frac{m f(y)}{(1-t)^{s}} .
$$

Remark 3. Let $\psi(x)=\frac{1}{x}, x \in(0, \infty)$. Then $\psi^{-1}(x)=\frac{1}{x}$, one can have the following inequality

$$
f\left((t x+m(1-t) y)^{-1}\right) \leq h\left(t^{\alpha}\right) f\left(\frac{1}{x}\right)+m h\left(1-t^{\alpha}\right) f\left(\frac{1}{y}\right) .
$$

By replacing $x$ with $\frac{1}{x}$ and $y$ with $\frac{1}{y}$ in (16), the definition of $(\alpha, h-m)$-HA-convexity defined in [33] can be obtained. Further, replacing $x$ with $\frac{1}{x}, y$ with $\frac{1}{y}$ and taking $\alpha=1$ in (16), the definition of $(\alpha, m)$-HA-convexity defined in [19] can be obtained. Similarly, replacing $x$ with $\frac{1}{x}, y$ with $\frac{1}{y}$ and taking $\alpha=m=1, h(t)=t$ in (16), definition of harmonic convexity defined in [8] can be obtained. Replacing $x$ with $\frac{1}{x}, y$ with $\frac{1}{y}$ and taking $\alpha=m=1, h(t)=t^{s}$ in (16), definition of harmonic $s$-convexity in the second sense defined in [32] can be obtained. Moreover, one can obtain harmonically $h$-convexity by replacing $x$ with $\frac{1}{x}, y$ with $\frac{1}{y}$ and taking $\alpha=m=1$ in (16) defined in [38] and s-Godunova-Levin function of second kind defined in [38] can be obtained by replacing $x$ with $\frac{1}{x}, y$ with $\frac{1}{y}$ and taking $\alpha=m=1, h(t)=t^{-s}$ in (16).

Remark 4. If $\alpha=m=1$ and $h(t)=t$ in (11), the definition of convexity with respect to a strictly monotone function stated in [3] can be obtained.

Remark 5. If $\alpha=m=1, \psi(t)=t, x=E(x), y=E(y), E: \mathbb{R} \rightarrow \mathbb{R}$ in (11), the definition of $h-E$-convexity stated in [39] can be obtained. If $\alpha=m=1, h(t)=\psi(t)=t, x=E(x)$, $y=E(y), E: \mathbb{R} \rightarrow \mathbb{R}$ in (11), the definition of E-convexity stated in [40] can be obtained.

Remark 6. If we replace $x$ with $\psi(x)$ and $y$ with $\psi(y)$, the inequality (11) becomes:

$$
f \circ \psi^{-1}(t \psi(x)+m(1-t) \psi(y)) \leq h\left(t^{\alpha}\right) f(x)+m h\left(1-t^{\alpha}\right) f(y) .
$$

Remark 7. If $\alpha=1$ in (11), the definition of $(h-m)$-convexity of $f$ with respect to to $\psi$ can be obtained, as given below:

$$
f \circ \psi^{-1}(t \psi(x)+m(1-t) \psi(y)) \leq h(t) f(x)+m h(1-t) f(y) .
$$

Remark 8. If $\alpha=1$ and $h(t)=t^{s}$ in (11), the definition of $(s, m)$-convexity of $f$ with respect to to strictly monotonic function $\psi$ can be obtained, as given below:

$$
f \circ \psi^{-1}(t \psi(x)+m(1-t) \psi(y)) \leq t^{s} f(x)+m(1-t)^{s} f(y) .
$$

Remark 9. If $h(t)=t$ in (11), the definition of $(\alpha, m)$-convexity of $f$ with respect to to $\psi$ can be obtained as given below:

$$
f \circ \psi^{-1}(t \psi(x)+m(1-t) \psi(y)) \leq t^{\alpha} f(x)+m\left(1-t^{\alpha}\right) f(y) .
$$


Remark 10. If $\alpha=1$ and $h(t)=t^{-s}$ in (11), the definition of Godunova-Levin type of $(s, m)$ convexity of $f$ with respect to to $\psi$ can be obtained as given below:

$$
f \circ \psi^{-1}(t \psi(x)+m(1-t) \psi(y)) \leq t^{-s} f(x)+m(1-t)^{-s} f(y) .
$$

3. Fractional Versions of Hadamard Inequalities for $(\alpha, h-m)$-Convex Function with Respect to Strictly Monotone Function

In this section, we have proved two variants of Hadamard inequalities for $(\alpha, h-m)$ convex functions with respect to strictly monotone function. The first one is stated and proved as follows:

Theorem 3. Let $I$, J be intervals in $\mathbb{R}$ and $f: I \rightarrow \mathbb{R}$ be the $(\alpha, h-m)$-convex function, also let $\psi: J \rightarrow \mathbb{R}$ be strictly monotonic function and $J \subset I, a, b \in J, f \in L_{1}[a, b]$. If $f$ is $(\alpha, h-m)$ convex function with respect to $\psi$, then for $(\alpha, m) \in(0,1]^{2}$ one can have the following inequality:

$$
\begin{aligned}
& f\left(\psi^{-1}\left(\frac{\psi(a)+m \psi(b)}{2}\right)\right) \leq \frac{\Gamma(\mu+1)}{(m \psi(b)-\psi(a))^{\mu}}\left[h\left(\frac{1}{2^{\alpha}}\right) I_{(\psi(a))^{+}}^{\mu} f\left(\psi^{-1}(m \psi(b))\right)\right. \\
& \left.+m^{\mu+1} H\left(\frac{1}{2}\right) I_{(\psi(b))^{-}}^{\mu} f\left(\psi^{-1}\left(\frac{\psi(a)}{m}\right)\right)\right] \leq \mu\left[h\left(\frac{1}{2^{\alpha}}\right) f(a)+m H\left(\frac{1}{2}\right) f(b)\right] \\
& \times \int_{0}^{1} h\left(t^{\alpha}\right) t^{\mu-1} d t+m \mu\left[h\left(\frac{1}{2^{\alpha}}\right) f(b)+m H\left(\frac{1}{2}\right) f\left(\psi^{-1}\left(\frac{\psi(a)}{m^{2}}\right)\right)\right] \int_{0}^{1} H(t) t^{\mu-1} d t,
\end{aligned}
$$

with $\mu>0$ and $H(t)=h\left(1-t^{\alpha}\right)$, provided $\frac{\psi(a)}{m}, \frac{\psi(a)}{m^{2}}, t x+m(1-t) y \in \operatorname{Im}(\psi)$ for $x, y \in \operatorname{Im}(\psi)$.

Proof. Since $f$ is $(\alpha, h-m)$-convex with respect to $\psi$, for $x, y \in \operatorname{Im}(\psi)$, the following inequality holds:

$$
f\left(\psi^{-1}\left(\frac{x+m y}{2}\right)\right) \leq h\left(\frac{1}{2^{\alpha}}\right) f\left(\psi^{-1}(x)\right)+m H\left(\frac{1}{2}\right) f\left(\psi^{-1}(y)\right) .
$$

Taking $x=t \psi(a)+m(1-t) \psi(b), y=\frac{\psi(a)}{m}(1-t)+\psi(b) t, t \in[0,1]$ in (23), we get the following inequality:

$$
\begin{aligned}
& f\left(\psi^{-1}\left(\frac{\psi(a)+m \psi(b)}{2}\right)\right) \leq h\left(\frac{1}{2^{\alpha}}\right) f\left(\psi^{-1}(t \psi(a) t+m(1-t) \psi(b))\right) \\
& +m H\left(\frac{1}{2}\right) f\left(\psi^{-1}\left(\frac{\psi(a)}{m}(1-t)+\psi(b) t\right)^{\frac{1}{p}}\right)
\end{aligned}
$$

Using $(\alpha, h-m)$-convexity of $f$ with respect to $\psi$ and integrating the resulting inequality over the interval $[0,1]$ after multiplying with $t^{\mu-1}$, we get:

$$
\begin{aligned}
& \frac{1}{\mu} f\left(\psi^{-1}\left(\frac{\psi(a)+m \psi(b)}{2}\right)\right) \leq h\left(\frac{1}{2^{\alpha}}\right) \int_{0}^{1} f\left(\psi^{-1}(\psi(a) t+m(1-t) \psi(b))\right) t^{\mu-1} d t+m H\left(\frac{1}{2}\right) \\
& \times \int_{0}^{1} f\left(\psi^{-1}\left(\frac{\psi(a)}{m}(1-t)+\psi(b) t\right)\right) t^{\mu-1} d t \leq\left[h\left(\frac{1}{2^{\alpha}}\right) f(a)+m H\left(\frac{1}{2}\right) f(b)\right] \int_{0}^{1} h\left(t^{\alpha}\right) t^{\mu-1} d t \\
& +m\left[h\left(\frac{1}{2^{\alpha}}\right) f(b)+m H\left(\frac{1}{2}\right) f\left(\psi^{-1}\left(\frac{\psi(a)}{m^{2}}\right)\right)\right] \int_{0}^{1} H(t) t^{\mu-1} d t .
\end{aligned}
$$

Taking $u=t \psi(a)+m(1-t) \psi(b)$, that is, $t=\frac{m \psi(b)-u}{m \psi(b)-\psi(a)}$ and $v=\frac{\psi(a)}{m}(1-t)+\psi(b) t$, that is, $t=\frac{v-\frac{\psi(a)}{m}}{\psi(b)-\frac{\psi(a)}{m}}$ in (25), then by applying Definition 2 and multiplying by $\mu$, the required inequality (22) can be obtained.

Remark 11. (i) If one considers $\psi(x)=\frac{1}{x}, h(t)=t, m=\alpha=1$ in (22), we obtain Theorem 4 from [9]. 
(ii) If one considers $\psi(x)=\frac{1}{x}, h(t)=t$ and $\alpha=\mu=m=1$ in (22), we obtain Theorem 2.4 from [8].

(iii) If one considers $\alpha=1$ and $\psi(t)=t$ in (22), we obtain Corollary 2.2 from [1].

(iv) If one considers $h(t)=\psi(t)=t$ and $m=\alpha=1$ in (22), we obtain Theorem 1.

(v) If one considers $h(t)=\psi(t)=t$ and $\alpha=\mu=m=1$ in (22), we obtain the Hadamard inequality.

(vi) If one considers $h(t)=\psi(t)=t$ and $\alpha=1$ in (22), we obtain Theorem 2.1 from [27].

(vii) If one considers $h(t)=t^{s}, \alpha=\mu=m=1$ and $\psi(x)=\frac{1}{x}$ in (22), we obtain Theorem 2.1 from [32].

(viii) If one considers $h(t)=t^{s}, \alpha=\mu=m=1$ and $\psi(x)=\frac{1}{x}$ in (22), we obtain Theorem 2.1 from [4].

(ix) If one considers $h(t)=t, \alpha=\mu=m=1$ and $\psi(x)=\ln x$ in (22), we obtain Theorem 7 from [7].

(x) If one considers $\alpha=\mu=m=1, \psi(x)=\ln x$ and $h(t)=t^{s}$ in (22), we obtain Corollary 2.1 from [31].

Corollary 1. The following fractional integral inequality holds for $(\alpha, h-m)$-GA-convex functions:

$$
\begin{aligned}
& f\left(\sqrt{a b^{m}}\right) \leq \frac{\Gamma(\mu+1)}{(m \ln b-\ln a)^{\mu}}\left[h\left(\frac{1}{2^{\alpha}}\right) I_{(\ln a)^{+}}^{\mu} f\left(b^{m}\right)+m^{\mu+1} H\left(\frac{1}{2}\right) I_{(\ln b)^{-}}^{\mu} f\left(a^{\frac{1}{m}}\right)\right] \\
& \leq \mu\left[h\left(\frac{1}{2^{\alpha}}\right) f(a)+m H\left(\frac{1}{2}\right) f(b)\right] \int_{0}^{1} h\left(t^{\alpha}\right) t^{\mu-1} d t+m \mu\left[h\left(\frac{1}{2^{\alpha}}\right) f(b)+m H\left(\frac{1}{2}\right) f\left(a^{\frac{1}{m^{2}}}\right)\right] \\
& \times \int_{0}^{1} H(t) t^{\mu-1} d t,
\end{aligned}
$$

provided $0<a<b$.

Proof. The function $\psi(x)=\ln x, x \in(0, \infty)$ is strictly increasing. By setting it in (22), we get above inequality.

Corollary 2. The following Riemann-Liouville fractional integral inequality for $(\alpha, h-m)-H A$ convex functions holds:

$$
\begin{aligned}
& f\left(\frac{2 a b}{a m+b}\right) \leq \frac{(a b)^{\mu} \Gamma(\mu+1)}{(a m-b)^{\mu}}\left[h\left(\frac{1}{2^{\alpha}}\right) I_{\left(\frac{1}{a}\right)^{+}}^{\mu} f\left(\frac{b}{m}\right)+m^{\mu+1} H\left(\frac{1}{2}\right) I_{\left(\frac{1}{b}\right)}^{\mu} f\left(\frac{1}{a m}\right)\right] \\
& \leq \mu\left[H\left(\frac{1}{2^{\alpha}}\right) f(a)+m H\left(\frac{1}{2}\right) f(b)\right] \int_{0}^{1} h\left(t^{\alpha}\right) t^{\mu-1} d t+m \mu\left[H\left(\frac{1}{2^{\alpha}}\right) f(b)+m H\left(\frac{1}{2}\right) f\left(a m^{2}\right)\right] \\
& \times \int_{0}^{1} H(t) t^{\mu-1} d t
\end{aligned}
$$

provided $0<a<b$.

Proof. The function $\psi(x)=\frac{1}{x}$ is strictly decreasing. By setting it in (22), we get above inequality.

Corollary 3. The following Riemann-Liouville fractional integral inequality for $(\alpha, m)$-convexity of $f$ with respect to a strictly monotonic function $\psi$ holds:

$$
\begin{aligned}
& f\left(\psi^{-1}\left(\frac{\psi(a)+m \psi(b)}{2}\right)\right) \leq \frac{\Gamma(\mu+1)}{2^{\alpha}(m \psi(b)-\psi(a))^{\mu}}\left[I_{(\psi(a))^{+}}^{\mu} f\left(\psi^{-1}(m \psi(b))\right)+m^{\mu+1}\right. \\
& \left.\times\left(2^{\alpha}-1\right) I_{(\psi(b))^{-}}^{\mu} f\left(\psi^{-1}\left(\frac{\psi(a)}{m}\right)\right)\right] \leq \frac{\mu\left[f(a)+m\left(2^{\alpha}-1\right) f(b)\right]}{2^{\alpha}(\alpha+\mu)}+\frac{m \alpha}{2^{\alpha}(\mu+\alpha)}[f(b) \\
& \left.+m\left(2^{\alpha}-1\right) f\left(\psi^{-1}\left(\frac{\psi(a)}{m^{2}}\right)\right)\right]
\end{aligned}
$$

provided $0<a<b$. 
Proof. For $h(t)=t$, we have $H(t)=1-t^{\alpha}$ and $\int_{0}^{1} h\left(t^{\alpha}\right) t^{\mu-1} d t=\int_{0}^{1} t^{\alpha+\mu-1} d t=\frac{1}{\alpha+\mu}$, $\int_{0}^{1} H(t) t^{\mu-1} d t=\int_{0}^{1}\left(t^{\mu-1}-t^{\alpha+\mu-1}\right) d t=\frac{\alpha}{\mu(\alpha+\mu)}$. Therefore from (22), the required inequality can be obtained.

Corollary 4. The following Riemann-Liouville fractional integral inequality for $(s, m)$-convexity of $f$ with respect to a strictly monotonic function $\psi$ holds:

$$
\begin{aligned}
& f\left(\psi^{-1}\left(\frac{\psi(a)+m \psi(b)}{2}\right)\right) \leq \frac{\Gamma(\mu+1)}{2^{s}(m \psi(b)-\psi(a))^{\mu}}\left[I_{(\psi(a))^{+}}^{\mu} f\left(\psi^{-1}(m \psi(b))\right)+m^{\mu+1}\right. \\
& \left.\times I_{(\psi(b))^{-}}^{\mu} f\left(\psi^{-1}\left(\frac{\psi(a)}{m}\right)\right)\right] \leq \frac{\mu[f(a)+m f(b)]}{2^{s}(s+\mu)}+\frac{m \mu B(1+s, \mu)}{2^{s}}\left[f(b)+m f\left(\psi^{-1}\left(\frac{\psi(a)}{m^{2}}\right)\right)\right] .
\end{aligned}
$$

Proof. If $\alpha=1$ and $h(t)=t^{s}$ in (22), then the above inequality can be obtained.

Corollary 5. The following Riemann-Liouville fractional integral inequality for Godunova-Levin type of $(s, m)$-convexity of $f$ with respect to a strictly monotonic function $\psi$ holds:

$$
\begin{aligned}
& f\left(\psi^{-1}\left(\frac{\psi(a)+m \psi(b)}{2}\right)\right) \leq \frac{2^{s} \Gamma(\mu+1)}{(m \psi(b)-\psi(a))^{\mu}}\left[I_{(\psi(a))^{+}}^{\mu} f\left(\psi^{-1}(m \psi(b))\right)+m^{\mu+1}\right. \\
& \left.\times I_{(\psi(b))^{-}}^{\mu} f\left(\psi^{-1}\left(\frac{\psi(a)}{m}\right)\right)\right] \leq \frac{2^{s} \mu[f(a)+m f(b)]}{s+\mu}+2^{s} m \mu B(1+s, \mu)\left[f(b)+m f\left(\psi^{-1}\left(\frac{\psi(a)}{m^{2}}\right)\right)\right] .
\end{aligned}
$$

Proof. If $\alpha=1$ and $h(t)=t^{-s}$ in (22), then the above inequality can be obtained.

Corollary 6. The following Riemann-Liouville fractional integral inequalities for $(\alpha, h-m)$-pconvex functions hold:

(i) If $p>0$,

$$
\begin{aligned}
& f\left(\left(\frac{a^{p}+m b^{p}}{2}\right)^{\frac{1}{p}}\right) \leq \frac{\Gamma(\mu+1)}{\left(m b^{p}-a^{p}\right)^{\mu}}\left[h\left(\frac{1}{2^{\alpha}}\right) I_{\left(a^{p}\right)^{+}}^{\mu} f\left(\left(m b^{p}\right)^{\frac{1}{p}}\right)+m^{\mu+1} H\left(\frac{1}{2}\right) I_{\left(b^{p}\right)^{-}}^{\mu} f\left(\left(\frac{a^{p}}{m}\right)^{\frac{1}{p}}\right)\right] \\
& \leq \mu\left[h\left(\frac{1}{2^{\alpha}}\right) f(a)+m H\left(\frac{1}{2}\right) f(b)\right] \int_{0}^{1} h\left(t^{\alpha}\right) t^{\mu-1} d t+m \mu\left[h\left(\frac{1}{2^{\alpha}}\right) f(b)+m H\left(\frac{1}{2}\right) f\left(\left(\frac{a^{p}}{m^{2}}\right)^{\frac{1}{p}}\right)\right] \\
& \times \int_{0}^{1} H(t) t^{\mu-1} d t .
\end{aligned}
$$

(ii) If $p<0$,

$$
\begin{aligned}
& f\left(\left(\frac{a^{p}+m b^{p}}{2}\right)^{\frac{1}{p}}\right) \leq \frac{\Gamma(\mu+1)}{\left(a^{p}-m b^{p}\right)^{\mu}}\left[h\left(\frac{1}{2^{\alpha}}\right) I_{\left(a^{p}\right)^{-}}^{\mu} f\left(\left(m b^{p}\right)^{\frac{1}{p}}\right)+m^{\mu+1} H\left(\frac{1}{2}\right) I_{\left(b^{p}\right)^{+}}^{\mu} f\left(\left(\frac{a^{p}}{m}\right)^{\frac{1}{p}}\right)\right] \\
& \leq \mu\left[h\left(\frac{1}{2^{\alpha}}\right) f(a)+m H\left(\frac{1}{2}\right) f(b)\right] \int_{0}^{1} h\left(t^{\alpha}\right) t^{\mu-1} d t+m \mu\left[h\left(\frac{1}{2^{\alpha}}\right) f(b)+m H\left(\frac{1}{2}\right) f\left(\left(\frac{a^{p}}{m^{2}}\right)^{\frac{1}{p}}\right)\right] \\
& \times \int_{0}^{1} H(t) t^{\mu-1} d t,
\end{aligned}
$$

provided $0<a<b$.

Proof. (i) The function $\psi(x)=x^{p}$ is strictly increasing on $(0, \infty)$ for $p>0$. By setting it in (22), we get the required inequality.

(ii) The function $\psi(x)=x^{p}$ is strictly decreasing on $(0, \infty)$ for $p<0$. By setting it in (22), we get the required inequality.

Remark 12. (i) If $\alpha=1$ in (26) and (27), then the inequalities for $(h-m)$-p-convex function can be obtained.

(ii) If one considers $\alpha=m=\mu=1$ and $h(t)=t$ in (26) and (27), we obtain Theorem 1.3 from [10]. 
Corollary 7. The following Riemann-Liouville fractional integral inequalities for $(s, m)$-p-GodunovaLevin functions hold:

(i) If $p>0$,

$$
\begin{aligned}
& f\left(\left(\frac{a^{p}+m b^{p}}{2}\right)^{\frac{1}{p}}\right) \leq \frac{2^{s} \Gamma(\mu+1)}{\left(m b^{p}-a^{p}\right)^{\mu}}\left[I_{\left(a^{p}\right)^{+}}^{\mu} f\left(\left(m b^{p}\right)^{\frac{1}{p}}\right)+m^{\mu+1} I_{\left(b^{p}\right)^{-}}^{\mu} f\left(\left(\frac{a^{p}}{m}\right)^{\frac{1}{p}}\right)\right] \\
& \leq \frac{2^{s} \mu[f(a)+m f(b)]}{\mu-s}+2^{s} m \mu B(\mu, 1-s)\left[f(b)+m f\left(\left(\frac{a^{p}}{m^{2}}\right)^{\frac{1}{p}}\right)\right] .
\end{aligned}
$$

(ii) If $p<0$,

$$
\begin{aligned}
& f\left(\left(\frac{a^{p}+m b^{p}}{2}\right)^{\frac{1}{p}}\right) \leq \frac{2^{s} \Gamma(\mu+1)}{\left(a^{p}-m b^{p}\right)^{\mu}}\left[I_{\left(a^{p}\right)^{-}}^{\mu} f\left(\left(m b^{p}\right)^{\frac{1}{p}}\right)+m^{\mu+1} I_{\left(b^{p}\right)^{+}}^{\mu} f\left(\left(\frac{a^{p}}{m}\right)^{\frac{1}{p}}\right)\right] \\
& \leq \frac{2^{s} \mu[f(a)+m f(b)]}{\mu-s}+2^{s} m \mu B(\mu, 1-s)\left[f(b)+m f\left(\left(\frac{a^{p}}{m^{2}}\right)^{\frac{1}{p}}\right)\right],
\end{aligned}
$$

provided $0<a<b$.

Proof. (i) If $\alpha=1$ and $h(t)=t^{-s}$ in (26), then the required inequality can be obtained. (ii) If $\alpha=1$ and $h(t)=t^{-s}$ in (27), then the required inequality can be obtained.

Corollary 8. The following Riemann-Liouville fractional integral inequalities for $(s, m)$-p-convex functions hold:

(i) If $p>0$,

$$
\begin{aligned}
& f\left(\left(\frac{a^{p}+m b^{p}}{2}\right)^{\frac{1}{p}}\right) \leq \frac{\Gamma(\mu+1)}{2^{s}\left(m b^{p}-a^{p}\right)^{\mu}}\left[I_{\left.\left(a^{p}\right)\right)^{+}}^{\mu} f\left(\left(m b^{p}\right)^{\frac{1}{p}}\right)+m^{\mu+1} I_{\left(b^{p}\right)^{-}}^{\mu} f\left(\left(\frac{a^{p}}{m}\right)^{\frac{1}{p}}\right)\right] \\
& \leq \frac{\mu[f(a)+m f(b)]}{2^{s}(\mu+s)}+\frac{m \mu B(\mu, 1+s)}{2^{s}}\left[f(b)+m f\left(\left(\frac{a^{p}}{m^{2}}\right)^{\frac{1}{p}}\right)\right] .
\end{aligned}
$$

(ii) If $p<0$,

$$
\begin{aligned}
& f\left(\left(\frac{a^{p}+m b^{p}}{2}\right)^{\frac{1}{p}}\right) \leq \frac{\Gamma(\mu+1)}{2^{s}\left(a^{p}-m b^{p}\right)^{\mu}}\left[I_{\left.\left(a^{p}\right)\right)^{-}}^{\mu} f\left(\left(m b^{p}\right)^{\frac{1}{p}}\right)+m^{\mu+1} I_{\left(b^{p}\right)^{+}}^{\mu} f\left(\left(\frac{a^{p}}{m}\right)^{\frac{1}{p}}\right)\right] \\
& \leq \frac{\mu[f(a)+m f(b)]}{2^{s}(\mu+s)}+\frac{m \mu B(\mu, 1+s)}{2^{s}}\left[f(b)+m f\left(\left(\frac{a^{p}}{m^{2}}\right)^{\frac{1}{p}}\right)\right],
\end{aligned}
$$

provided $0<a<b$.

Proof. (i) If $\alpha=1$ and $h(t)=t^{s}$ in (26), then the required inequality can be obtained. (ii) If $\alpha=1$ and $h(t)=t^{s}$ in (27), then the required inequality can be obtained.

Corollary 9. The following Riemann-Liouville fractional integral inequalities for $(\alpha, m)$-p-convex functions hold:

(i) If $p>0$,

$$
\begin{aligned}
& f\left(\left(\frac{a^{p}+m b^{p}}{2}\right)^{\frac{1}{p}}\right) \leq \frac{\Gamma(\mu+1)}{2^{\alpha}\left(m b^{p}-a^{p}\right)^{\mu}}\left[I_{\left(a^{p}\right)^{+}}^{\mu} f\left(\left(m b^{p}\right)^{\frac{1}{p}}\right)+m^{\mu+1}\left(2^{\alpha}-1\right) I_{\left(b^{p}\right)^{-}}^{\mu} f\left(\left(\frac{a^{p}}{m}\right)^{\frac{1}{p}}\right)\right] \\
& \leq \frac{\mu\left[f(a)+m\left(2^{\alpha}-1\right) f(b)\right]}{2^{\alpha}(\mu+\alpha)}+\frac{m \alpha}{2^{\alpha}(\mu+\alpha)}\left[f(b)+m f\left(\left(\frac{a^{p}}{m^{2}}\right)^{\frac{1}{p}}\right)\right] .
\end{aligned}
$$


(ii) If $p<0$,

$$
\begin{aligned}
& f\left(\left(\frac{a^{p}+m b^{p}}{2}\right)^{\frac{1}{p}}\right) \leq \frac{\Gamma(\mu+1)}{2^{\alpha}\left(a^{p}-m b^{p}\right)^{\mu}}\left[I_{\left(a^{p}\right)^{-}}^{\mu} f\left(\left(m b^{p}\right)^{\frac{1}{p}}\right)+m^{\mu+1}\left(2^{\alpha}-1\right) I_{\left(b^{p}\right)^{+}}^{\mu} f\left(\left(\frac{a^{p}}{m}\right)^{\frac{1}{p}}\right)\right] \\
& \leq \frac{\mu\left[f(a)+m\left(2^{\alpha}-1\right) f(b)\right]}{2^{\alpha}(\mu+\alpha)}+\frac{m \alpha}{2^{\alpha}(\mu+\alpha)}\left[f(b)+m f\left(\left(\frac{a^{p}}{m^{2}}\right)^{\frac{1}{p}}\right)\right],
\end{aligned}
$$

provided $0<a<b$.

Proof. (i) If $h(t)=t$ in (26), then the required inequality can be obtained.

(ii) If $h(t)=t$ in (27), then the required inequality can be obtained.

Corollary 10. The following Riemann-Liouville fractional integral inequality for $(\alpha, h-m)-H A$ convex functions holds:

$$
\begin{aligned}
& f\left(\frac{2 a b}{b+a m}\right) \leq \frac{\Gamma(\mu+1)(a b)^{\mu}}{(b-m a)^{\mu}}\left[h\left(\frac{1}{2^{\alpha}}\right) I_{\left(\frac{1}{a}\right)^{-}}^{\mu} f\left(\frac{b}{m}\right)+m^{\mu+1} H\left(\frac{1}{2}\right) I_{\left(\frac{1}{b}\right)^{+}}^{\mu} f(m a)\right] \\
& \leq \mu\left[h\left(\frac{1}{2^{\alpha}}\right) f(a)+m H\left(\frac{1}{2}\right) f(b)\right] \int_{0}^{1} h\left(t^{\alpha}\right) t^{\mu-1} d t+m \mu\left[h\left(\frac{1}{2^{\alpha}}\right) f(b)+m H\left(\frac{1}{2}\right) f\left(m^{2} a\right)\right] \\
& \times \int_{0}^{1} H(t) t^{\mu-1} d t .
\end{aligned}
$$

Proof. If $p=-1$ in (27), then the above inequality can be obtained.

The second variant of the Hadamard inequality is stated and proved as follows:

Theorem 4. Under the same assumptions as stated in Theorem 3, for Riemann-Liouville fractional integrals the following inequality holds:

$$
\begin{aligned}
& f\left(\psi^{-1}\left(\frac{\psi(a)+m \psi(b)}{2}\right)\right) \leq \frac{2^{\mu} \Gamma(\mu+1)}{(m \psi(b)-\psi(a))^{\mu}}\left[h\left(\frac{1}{2^{\alpha}}\right) I^{\mu}\left(\frac{\psi(a)+m \psi(b)}{2}\right)^{+} f\left(\psi^{-1}(m \psi(b))\right)\right. \\
& \left.+m^{\mu+1} H\left(\frac{1}{2}\right) I_{\left(\frac{\psi(a)+m \psi(b)}{2 m}\right)}^{\mu} f\left(\psi^{-1}\left(\frac{\psi(a)}{m}\right)\right)\right] \leq \mu\left[h\left(\frac{1}{2^{\alpha}}\right) f(a)+m H\left(\frac{1}{2}\right) f(b)\right] \\
& \times \int_{0}^{1} h\left(\frac{t^{\alpha}}{2^{\alpha}}\right) t^{\mu-1} d t+m \mu\left[h\left(\frac{1}{2^{\alpha}}\right) f(b)+m H\left(\frac{1}{2}\right) f\left(\psi^{-1}\left(\frac{\psi(a)}{m^{2}}\right)\right)\right] \int_{0}^{1} H\left(\frac{t}{2}\right) t^{\mu-1} d t .
\end{aligned}
$$

Proof. Let $x=\frac{\psi(a) t}{2}+m\left(\frac{2-t}{2}\right) \psi(b), y=\frac{\psi(a)}{m}\left(\frac{2-t}{2}\right)+\frac{\psi(b) t}{2}$ in (23), we get:

$$
\begin{aligned}
& f\left(\psi^{-1}\left(\frac{\psi(a)+m \psi(b)}{2}\right)\right) \leq h\left(\frac{1}{2^{\alpha}}\right) f\left(\psi^{-1}\left(\frac{\psi(a) t}{2}+m\left(\frac{2-t}{2}\right) \psi(b)\right)\right) \\
& +m H\left(\frac{1}{2}\right) f\left(\psi^{-1}\left(\frac{\psi(a)}{m}\left(\frac{2-t}{2}\right)+\frac{\psi(a) t}{2}\right)\right) .
\end{aligned}
$$

Using $(\alpha, h-m)$-convexity of $f$ with respect to $\psi$ and integrating the resulting inequality over the interval $[0,1]$ after multiplying with $t^{\mu-1}$, we get:

$$
\begin{aligned}
& \frac{1}{\mu} f\left(\psi^{-1}\left(\frac{\psi(a)+m \psi(b)}{2}\right)\right) \leq h\left(\frac{1}{2^{\alpha}}\right) \int_{0}^{1} f\left(\psi^{-1}\left(\frac{\psi(a) t}{2}+m\left(\frac{2-t}{2}\right) \psi(b)\right)\right) t^{\mu-1} d t \\
& \left.+m H\left(\frac{1}{2}\right) \int_{0}^{1} f\left(\psi^{-1}\left(\frac{\psi(a)}{m}\left(\frac{2-t}{2}\right)+\frac{\psi(a) t}{2}\right)\right) t^{\mu-1} d t\right] \leq\left[h\left(\frac{1}{2^{\alpha}}\right) f(a)+m H\left(\frac{1}{2}\right) f(b)\right] \\
& \times \int_{0}^{1} h\left(\frac{t^{\alpha}}{2^{\alpha}}\right) t^{\mu-1} d t+m\left[h\left(\frac{1}{2^{\alpha}}\right) f(b)+m H\left(\frac{1}{2}\right) f\left(\frac{\psi(a)}{m^{2}}\right)\right] \int_{0}^{1} H\left(\frac{t}{2}\right) t^{\mu-1} d t .
\end{aligned}
$$


Taking $u=\frac{\psi(a) t}{2}+m\left(\frac{2-t}{2}\right)(b)$, that is, $t=\frac{2(m \psi(b)-u)}{m \psi(b)-\psi(a)}$ and $v=\frac{\psi(a)}{m}\left(\frac{2-t}{2}\right)+\frac{\psi(b) t}{2}$, that is, $t=\frac{2\left(v-\frac{\psi(a)}{m}\right)}{\psi(b)-\frac{\psi(a)}{m}}$ in (30), then by applying Definition 2 and multiplying by $\mu$, we get the required inequality $(28)$.

Remark 13. (i) If one considers $\alpha=m=1$ and $h(t)=t=\psi(t)$ in (28), we obtain Theorem 2.

(ii) If one considers $h(t)=\psi(t)=t, \alpha=\mu=m=1$ in (28), we obtain the Hadamard inequality.

(iii) If one considers $h(t)=\psi(t)=t$ and $\alpha=1$ in (28), we obtain Theorem 2.1 from [28].

(iv) If one considers $h(t)=t, \alpha=m=1$ and $\psi(x)=\frac{1}{x}$ in (28), we obtain Theorem 4 from [11].

(v) If one considers $h(t)=t, \psi(x)=\frac{1}{x}$ and $\alpha=\mu=m=1$ in (28), we obtain Theorem 2.4 from [8].

(vi) If one considers $\psi(t)=t, \alpha=\mu=m=1$ and $h(t)=t^{-s}$ in (28), we obtain Theorem 7 from [29].

(vii) If one considers $h(t)=t^{-s}, \alpha=\mu=m=1$ and $\psi(x)=\frac{1}{x}$ in (28), we obtain Theorem 3.1 from [30].

(viii) If one considers $h(t)=t^{s}, \alpha=\mu=m=1$ and $\psi(x)=\frac{1}{x}$ in (28), we obtain Theorem 2.1 from [32].

(ix) If one considers $\alpha=\mu=m=1, h(t)=t^{s}$ and $\psi(x)=\frac{1}{x}$ in (28), we obtain Theorem 2.1 from [4].

Corollary 11. The following Riemann-Liouville fractional integral inequality for $(\alpha, h-m)-G A$ convex functions holds:

$$
\begin{aligned}
& f\left(\sqrt{a b^{m}}\right) \leq \frac{2^{\mu} \Gamma(\mu+1)}{(m \ln b-\ln a)^{\mu}}\left[h\left(\frac{1}{2^{\alpha}}\right) I_{\left(\frac{\ln a+m \ln b}{2}\right)^{+}}^{\mu} f\left(b^{m}\right)+m^{\mu+1} H\left(\frac{1}{2}\right) I_{\left(\frac{\ln a+m \ln b}{2 m}\right)^{-}}^{\mu} f\left(a^{m}\right)\right] \\
& \leq \mu\left[h\left(\frac{1}{2^{\alpha}}\right) f(a)+m H\left(\frac{1}{2}\right) f(b)\right] \int_{0}^{1} h\left(\frac{t^{\alpha}}{2^{\alpha}}\right) t^{\mu-1} d t+m \mu\left[h\left(\frac{1}{2^{\alpha}}\right) f(b)+m H\left(\frac{1}{2}\right) f\left(a^{m^{2}}\right)\right] \\
& \times \int_{0}^{1} H\left(\frac{t}{2}\right) t^{\mu-1} d t,
\end{aligned}
$$

provided $0<a<b$.

Proof. The function $\psi(x)=\ln x, x \in(0, \infty)$ is strictly increasing. By setting it in (28), we get above inequality.

Corollary 12. The following Riemann-Liouville fractional integral inequality for $(\alpha, h-m)-H A$ convex functions holds:

$$
\begin{aligned}
& f\left(\frac{2 a b}{a m+b}\right) \leq \frac{2^{\mu}(a b)^{\mu} \Gamma(\mu+1)}{(a m-b)^{\mu}}\left[h\left(\frac{1}{2^{\alpha}}\right) I_{\left(\frac{a m+b}{2 a b}\right)^{+}}^{\mu} f\left(b^{m}\right)+m^{\mu+1} H\left(\frac{1}{2}\right) I_{\left(\frac{a m+b}{2 m}\right)^{-}}^{\mu} f\left(a^{m}\right)\right] \\
& \leq \mu\left[h\left(\frac{1}{2^{\alpha}}\right) f(a)+m H\left(\frac{1}{2}\right) f(b)\right] \int_{0}^{1} h\left(\frac{t^{\alpha}}{2^{\alpha}}\right) t^{\mu-1} d t+m \mu\left[h\left(\frac{1}{2^{\alpha}}\right) f(b)+m H\left(\frac{1}{2}\right) f\left(a^{\frac{1}{m^{2}}}\right)\right] \\
& \times \int_{0}^{1} H\left(\frac{t}{2}\right) t^{\mu-1} d t
\end{aligned}
$$

provided $0<a<b$.

Proof. The function $\psi(x)=\frac{1}{x}, x \in(0, \infty)$ is strictly increasing. By setting it in (28), we get above inequality. 
Corollary 13. The following Riemann-Liouville fractional integral inequality for $(\alpha, m)$-convexity of $f$ with respect to a strictly monotonic function $\psi$ holds:

$$
\begin{aligned}
& f\left(\psi^{-1}\left(\frac{\psi(a)+m \psi(b)}{2}\right)\right) \leq \frac{2^{\mu-\alpha} \Gamma(\mu+1)}{(m \psi(b)-\psi(a))^{\mu}}\left[I_{\left(\frac{\psi(a)+m \psi(b)}{2}\right)^{+}}^{\mu} f\left(\psi^{-1}(m \psi(b))\right)+m^{\mu+1}\right. \\
& \left.\times\left(2^{\alpha}-1\right) I_{\left(\frac{\psi(a)+m \psi(b)}{2 m}\right)^{-}}^{\mu} f\left(\psi^{-1}\left(\frac{\psi(a)}{m}\right)\right)\right] \leq \frac{\mu\left[f(a)+m\left(2^{\alpha}-1\right) f(b)\right]}{2^{2 \alpha}(\mu+\alpha)} \\
& +\frac{m\left(2^{\alpha}(\mu+\alpha)-\mu\right)}{2^{2 \alpha}(\mu+\alpha)}\left[f(b)+m\left(2^{\alpha}-1\right) f\left(\psi^{-1}\left(\frac{\psi(a)}{m^{2}}\right)\right)\right] .
\end{aligned}
$$

Proof. If $h(t)=t$ in (28), then the above inequality can be obtained.

Corollary 14. The following Riemann-Liouville fractional integral inequality for Godunova Levin type of $(s, m)$-convexity of $f$ with respect to a strictly monotonic function $\psi$ holds:

$$
\begin{aligned}
& f\left(\psi^{-1}\left(\frac{\psi(a)+m \psi(b)}{2}\right)\right) \leq \frac{2^{\mu+s} \Gamma(\mu+1)}{(m \psi(b)-\psi(a))^{\mu}}\left[I_{\left(\frac{\psi(a)+m \psi(b)}{2}\right)^{\mu}}^{+} f\left(\psi^{-1}(m \psi(b))\right)+m^{\mu+1}\right. \\
& \left.\times I_{\left(\frac{\psi(a)+m \psi(b)}{2 m}\right)}^{\mu} f\left(\psi^{-1}\left(\frac{\psi(a)}{m}\right)\right)\right] \leq \frac{2^{2 s} \mu[f(a)+m f(b)]}{s+\mu}+2^{2 s} m \mu B\left(\frac{1}{2}, \mu, 1+s\right)[f(b) \\
& \left.+m f\left(\psi^{-1}\left(\frac{\psi(a)}{m^{2}}\right)\right)\right] \text {. }
\end{aligned}
$$

Proof. If $\alpha=1$ and $h(t)=t^{-s}$ in (28), then the above inequality can be obtained.

Corollary 15. The following Riemann-Liouville fractional integral inequality for $(s, m)$-convexity of $f$ with respect to a strictly monotonic function $\psi$ holds:

$$
\begin{aligned}
& f\left(\psi^{-1}\left(\frac{\psi(a)+m \psi(b)}{2}\right)\right) \leq \frac{2^{\mu-s} \Gamma(\mu+1)}{(m \psi(b)-\psi(a))^{\mu}}\left[I_{\left(\frac{\psi(a)+m \psi(b)}{2}\right)^{+}}^{\mu} f\left(\psi^{-1}(m \psi(b))\right)+m^{\mu+1}\right. \\
& \left.\times I_{\left(\frac{\psi(a)+m \psi(b)}{2 m}\right)}^{\mu} f\left(\psi^{-1}\left(\frac{\psi(a)}{m}\right)\right)\right] \leq \frac{\mu[f(a)+m f(b)]}{2^{2 s}(s+\mu)}+\frac{m \mu B\left(\frac{1}{2}, \mu, 1+s\right)}{2^{s}}[f(b) \\
& \left.+m f\left(\psi^{-1}\left(\frac{\psi(a)}{m^{2}}\right)\right)\right] .
\end{aligned}
$$

Proof. If $\alpha=1$ and $h(t)=t^{s}$ in (28), then the above inequality can be obtained.

Corollary 16. The following Riemann-Liouville fractional integral inequalities for $(\alpha, h-m)-p$ convex functions hold:

(i) If $p>0$,

$$
\begin{aligned}
& f\left(\left(\frac{a^{p}+m b^{p}}{2}\right)^{\frac{1}{p}}\right) \leq \frac{2^{\mu} \Gamma(\mu+1)}{\left(m b^{p}-a^{p}\right)^{\mu}}\left[h\left(\frac{1}{2^{\alpha}}\right) I_{\left(\frac{a^{p}+m b^{p}}{2}\right)}^{\mu} f\left(\left(m b^{p}\right)^{\frac{1}{p}}\right)+m^{\mu+1} H\left(\frac{1}{2}\right)\right. \\
& \left.\times I_{\left(\frac{a^{p}+m b^{p}}{2 m}\right)^{\mu}}^{\mu} f\left(\left(\frac{a^{p}}{m}\right)^{\frac{1}{p}}\right)\right] \leq \mu\left[h\left(\frac{1}{2^{\alpha}}\right) f(a)+m H\left(\frac{1}{2}\right) f(b)\right] \int_{0}^{1}\left(\frac{t^{\alpha}}{2^{\alpha}}\right) t^{\mu-1} d t \\
& +\mu m\left[h\left(\frac{1}{2^{\alpha}}\right) f(b)+m H\left(\frac{1}{2}\right) f\left(\left(\frac{a^{p}}{m^{2}}\right)^{\frac{1}{p}}\right)\right] \int_{0}^{1} H\left(\frac{t}{2}\right) t^{\mu-1} d t .
\end{aligned}
$$


(ii) If $p<0$,

$$
\begin{aligned}
& f\left(\left(\frac{a^{p}+m b^{p}}{2}\right)^{\frac{1}{p}}\right) \leq \frac{2^{\mu} \Gamma(\mu+1)}{\left(a^{p}-m b^{p}\right)^{\mu}}\left[h\left(\frac{1}{2^{\alpha}}\right) I_{\left(\frac{a^{p}+m b^{p}}{2}\right)^{-}}^{\mu} f\left(\left(m b^{p}\right)^{\frac{1}{p}}\right)+m^{\mu+1} H\left(\frac{1}{2}\right)\right. \\
& \left.\times I_{\left(\frac{a^{p}+m b p}{2 m}\right)}^{\mu} f\left(\left(\frac{a^{p}}{m}\right)^{\frac{1}{p}}\right)\right] \leq \mu\left[h\left(\frac{1}{2^{\alpha}}\right) f(a)+m H\left(\frac{1}{2}\right) f(b)\right] \int_{0}^{1}\left(\frac{t^{\alpha}}{2^{\alpha}}\right) t^{\mu-1} d t \\
& +\mu m\left[h\left(\frac{1}{2^{\alpha}}\right) f(b)+m H\left(\frac{1}{2}\right) f\left(\left(\frac{a^{p}}{m^{2}}\right)^{\frac{1}{p}}\right)\right] \int_{0}^{1} H\left(\frac{t}{2}\right) t^{\mu-1} d t,
\end{aligned}
$$

provided $0<a<b$.

Proof. (i) The function $\psi(x)=x^{p}$ is strictly increasing on $(0, \infty)$ for $p>0$. By setting it in (28), we get the required inequality.

(ii) The function $\psi(x)=x^{p}$ is strictly decreasing on $(0, \infty)$ for $p<0$. By setting it in (28), we get the required inequality.

Remark 14. (i) If $\alpha=1$ in (31) and (32), the inequalities for $(h-m)$-p-convex functions are obtained.

(ii) If one considers $\alpha=m=1$ and $h(t)=t$ in (31), the inequality (7) given in ([10], Theorem 7) is obtained.

(iii) If one considers $\alpha=m=1$ and $h(t)=t$ in (32), the inequality (8) given in ([10], Theorem 7) is obtained.

(iv) If one considers $\alpha=\mu=m=1, h(t)=t$ in (31) and (32), ([10], Theorem 1.3) is obtained.

Corollary 17. The following Riemann-Liouville fractional integral inequalities for $(s, m)$-pGodunova-Levin functions hold:

(i) If $p>0$,

$$
\begin{aligned}
& f\left(\left(\frac{a^{p}+m b^{p}}{2}\right)^{\frac{1}{p}}\right) \leq \frac{2^{\mu+s} \Gamma(\mu+1)}{\left(m b^{p}-a^{p}\right)^{\mu}}\left[I_{\left(\frac{a^{p}+m b^{p}}{2}\right)^{+}}^{\mu} f\left(\left(m b^{p}\right)^{\frac{1}{p}}\right)+m^{\mu+1} I_{\left(\frac{a^{p}+m b^{p}}{2 m}\right)^{\mu, \psi}}^{\mu} f\left(\left(\frac{a^{p}}{m}\right)^{\frac{1}{p}}\right)\right] \\
& \leq \frac{2^{2 s} \mu[f(a)+m f(b)]}{\mu-s}+2^{s} m_{2} F_{1}\left(s, \mu, 1+\mu, \frac{1}{2}\right)\left[f(b)+m f\left(\left(\frac{a^{p}}{m^{2}}\right)^{\frac{1}{p}}\right)\right] .
\end{aligned}
$$

(ii) If $p<0$,

$$
\begin{aligned}
& f\left(\left(\frac{a^{p}+m b^{p}}{2}\right)^{\frac{1}{p}}\right) \leq \frac{2^{\mu+s} \Gamma(\mu+1)}{\left(a^{p}-m b^{p}\right)^{\mu}}\left[I_{\left(\frac{a^{p}+m b^{p}}{2}\right)^{-}}^{\mu} f\left(\left(m b^{p}\right)^{\frac{1}{p}}\right)+m^{\mu+1} I_{\left(\frac{a^{p}+m b^{p}}{2 m}\right)^{+}}^{\mu} f\left(\left(\frac{a^{p}}{m}\right)^{\frac{1}{p}}\right)\right] \\
& \leq \frac{2^{2 s} \mu[f(a)+m f(b)]}{\mu-s}+2^{s} m_{2} F_{1}\left(s, \mu, 1+\mu, \frac{1}{2}\right)\left[f(b)+m f\left(\left(\frac{a^{p}}{m^{2}}\right)^{\frac{1}{p}}\right)\right],
\end{aligned}
$$

provided $0<a<b$.

Proof. (i) If $\alpha=1$ and $h(t)=t^{-s}$ in (31), then the required inequality can be obtained. (ii) If $\alpha=1$ and $h(t)=t^{-s}$ in (32), then the required inequality can be obtained. 
Corollary 18. The following Riemann-Liouville fractional integral inequalities for $(s, m)$-p-convex functions hold:

(i) If $p>0$,

$$
\begin{aligned}
& f\left(\left(\frac{a^{p}+m b^{p}}{2}\right)^{\frac{1}{p}}\right) \leq \frac{2^{\mu-s} \Gamma(\mu+1)}{\left(m b^{p}-a^{p}\right)^{\mu}}\left[I_{\left(\frac{a^{p}+m b p}{2}\right)^{+}}^{\mu} f\left(\left(m b^{p}\right)^{\frac{1}{p}}\right)+m^{\mu+1} I_{\left(\frac{a^{p}+m b p}{2 m}\right)^{-}}^{\mu} f\left(\left(\frac{a^{p}}{m}\right)^{\frac{1}{p}}\right)\right] \\
& \leq \frac{\mu[f(a)+m f(b)]}{2^{2 s}(\mu+s)}+2^{\mu-s} \mu m B\left(\frac{1}{2^{2}}, \mu, 1+s\right)\left[f(b)+m f\left(\frac{\psi(a)}{m^{2}}\right)\right] .
\end{aligned}
$$

(ii) If $p<0$,

$$
\begin{aligned}
& f\left(\left(\frac{a^{p}+m b^{p}}{2}\right)^{\frac{1}{p}}\right) \leq \frac{2^{\mu-s} \Gamma(\mu+1)}{\left(a^{p}-m b^{p}\right)^{\mu}}\left[I^{\mu}\left(\frac{a^{p}+m b^{p}}{2}\right)^{-} f\left(\left(m b^{p}\right)^{\frac{1}{p}}\right)+m^{\mu+1} I_{\left(\frac{a^{p}+m b p}{2 m}\right)^{\mu}}^{+f}\left(\left(\frac{a^{p}}{m}\right)^{\frac{1}{p}}\right)\right] \\
& \leq \frac{\mu[f(a)+m f(b)]}{2^{2 s}(\mu+s)}+2^{\mu-s} B\left(\frac{1}{2^{2}}, \mu, 1+s\right) \mu m\left[f(b)+m f\left(\frac{\psi(a)}{m^{2}}\right)\right],
\end{aligned}
$$

provided $0<a<b$.

Proof. (i) If $\alpha=1$ and $h(t)=t^{s}$ in (31), then the required inequality can be obtained.

(ii) If $\alpha=1$ and $h(t)=t^{s}$ in (32), then the required inequality can be obtained.

Corollary 19. The following Riemann-Liouville fractional integral inequalities for $(\alpha, m)$-pconvex functions hold:

(i) If $p>0$,

$$
\begin{aligned}
& f\left(\left(\frac{a^{p}+m b^{p}}{2}\right)^{\frac{1}{p}}\right) \leq \frac{2^{\mu-\alpha} \Gamma(\mu+1)}{\left(m b^{p}-a^{p}\right)^{\mu}}\left[I_{\left(\frac{a^{p}+m b^{p}}{2}\right)^{+}}^{\mu} f\left(\left(m b^{p}\right)^{\frac{1}{p}}\right)+m^{\mu+1}\left(2^{\alpha}-1\right)\right. \\
& \left.\times I_{\left(\frac{a^{p}+m b^{p}}{2 m}\right)^{-}}^{\mu} f\left(\left(\frac{a^{p}}{m}\right)^{\frac{1}{p}}\right)\right] \leq \frac{\mu\left[f(a)+m\left(2^{\alpha}-1\right) f(b)\right]}{2^{2 \alpha}(\mu+\alpha)}+\frac{m\left(2^{\alpha}(\mu+\alpha)-\mu\right)}{2^{2 \alpha}(\mu+\alpha)} \\
& \times\left[f(b)+m\left(2^{\alpha}-1\right) f\left(\left(\frac{a^{p}}{m^{2}}\right)^{\frac{1}{p}}\right)\right] .
\end{aligned}
$$

(ii) If $p<0$,

$$
\begin{aligned}
& f\left(\left(\frac{a^{p}+m b^{p}}{2}\right)^{\frac{1}{p}}\right) \leq \frac{2^{\mu-\alpha} \Gamma(\mu+1)}{\left(a^{p}-m b^{p}\right)^{\mu}}\left[I_{\left(\frac{a^{p}+m b^{p}}{2}\right)^{-}}^{\mu} f\left(\left(m b^{p}\right)^{\frac{1}{p}}\right)+m^{\mu+1}\left(2^{\alpha}-1\right)\right. \\
& \left.\times I_{\left(\frac{a^{p}+m b}{2 m}\right)^{+}} f\left(\left(\frac{a^{p}}{m}\right)^{\frac{1}{p}}\right)\right] \leq \frac{\mu\left[f(a)+m\left(2^{\alpha}-1\right) f(b)\right]}{2^{2 \alpha}(\mu+\alpha)}+\frac{m\left(2^{\alpha}(\mu+\alpha)-\mu\right)}{2^{2 \alpha}(\mu+\alpha)} \\
& \times\left[f(b)+m\left(2^{\alpha}-1\right) f\left(\left(\frac{a^{p}}{m^{2}}\right)^{\frac{1}{p}}\right)\right],
\end{aligned}
$$

provided $0<a<b$.

Proof. (i) If $h(t)=t$ in (31), then the required inequality is obtained.

(ii) If $h(t)=t$ in (32), then the required inequality can be obtained. 
Corollary 20. The following Riemann-Liouville fractional integral inequality for $(\alpha, h-m)-H A$ convex functions holds:

$$
\begin{aligned}
& f\left(\frac{2 a b}{b+m a}\right) \leq \frac{(2 a b)^{\mu} \Gamma(\mu+1)}{(b-m a)^{\mu}}\left[h\left(\frac{1}{2^{\alpha}}\right) I_{\left(\frac{b+m a}{2 a b}\right)^{-}}^{\mu} f\left(\frac{b}{m}\right)+m^{\mu+1} H\left(\frac{1}{2}\right) I_{\left(\frac{b+m a)}{2 m a b}\right)}^{\mu} f(m a)\right] \\
& \leq \mu\left[h\left(\frac{1}{2^{\alpha}}\right) f(a)+m H\left(\frac{1}{2}\right) f(b)\right] \int_{0}^{1} h\left(\frac{t^{\alpha}}{2^{\alpha}}\right) t^{\mu-1} d t+\mu m\left[h\left(\frac{1}{2^{\alpha}}\right) f(b)+m H\left(\frac{1}{2}\right) f\left(m^{2} a\right)\right] \\
& \times \int_{0}^{1} H\left(\frac{t}{2}\right) t^{\mu-1} d t
\end{aligned}
$$

Proof. If $p=-1$ in (32), then the above inequality can be obtained.

Remark 15. Using (9) and (10) after replacing $\mu$ by $\frac{\mu}{k}$ in all of the above inequalities, one can obtain $k$-fractional versions of all above results.

\section{Conclusions}

In this study, we have investigated a new type of convexity named as $(\alpha, h-m)$ convexity with respect to a strictly monotonic function that unifies several types of convexities, and proved some Hadamard type inequalities for this type of convexity via classical Riemann-Liouville fractional integrals. The outcomes of this paper provide the Hadamard inequalities for different types of convexities already established in the literature. This may be a leading step to obtain different results via other types of fractional integral operators. The $k$-fractional versions of Hadamard inequalities can also be obtained for newly introduced definition with the help of a parameter substitution.

Author Contributions: Conceptualization, T.Y., G.F., H.Y. and C.Y.J.; investigation, T.Y., G.F., H.Y. and C.Y.J.; methodology, T.Y., G.F., H.Y. and C.Y.J.; validation, T.Y., G.F., H.Y. and C.Y.J.; visualization, T.Y., G.F., H.Y. and C.Y.J.; writing-original draft, T.Y., G.F., H.Y. and C.Y.J.; writing-review and editing, T.Y., G.F., H.Y. and C.Y.J. All authors have read and agreed to the published version of the manuscript.

Funding: This research received no external funding.

Institutional Review Board Statement: Not applicable.

Informed Consent Statement: Not applicable.

Data Availability Statement: Not applicable.

Conflicts of Interest: The authors declare no conflict of interest.

\section{References}

1. Farid, G.; Rehman, A.U.; Ain, Q.U. $k$-fractional integral inequalities of Hadamard type for $(h-m)$-convex functions. Comput. Methods Differ. Equ. 2020, 8, 119-140.

2. Bermudo, S.; Korus, P.; Valdes, J.E.N. On q-Hermite-Hadamard inequalities for general convex functions. Acta Math. Hung. 2020, 162, 364-374. [CrossRef]

3. Dragomir, S.S. Inequalities of Hermite-Hadamard Type for composite convex Functions. In Frontiers in Functional Equations and Analytic Inequalities; Anastassiou, G., Rassias, J. Eds.; Springer: Cham, Switzerland, 2019; pp. 559-584.

4. Dragomir, S.S.; Fitzpatrick, S. The Hadamard inequalities for s-convex functions in the second sense. Demonstr. Math. 1999, 32, 687-696. [CrossRef]

5. Delavar, M.R.; Sen, M.D.L. Some generalizations of Hermite-Hadamard type inequalities. SpringerPlus 2016, 5, 1-9.

6. Farid, G.; Rehman, A.U.; Bibi, S.; Chu, Y.M. Refinements of two fractional versions of Hadamard inequalities for Caputo fractional derivatives and related results. Open J. Math. Sci. 2021, 5, 1-10. [CrossRef]

7. İşan, İ. Hermite-Hadamard type inequalities for GA-s-convex functions. Matematiche 2014, 69, 129-146.

8. İşan, İ. Hermite-Hadamard type inequalities for harmonically convex functions. Hacet. J. Math. Stat. 2014, 43, 935-942.

9. İşan, İ.; Wu, S. Hermite-Hadamard type inequalities for harmonically convex functions via fractional integrals. Appl. Math. Comput. 2014, 238, 237-244.

10. Kunt, M.; İşcan, İ. Hermite-Hadamard type inequalities for $p$-convex functions via fractional integrals. Moroccan J. Pure Appl. Anal. 2017, 3, 22-35. [CrossRef] 
11. Kunt, M.; İşcan, İ.; Yazici, N.; Gözütok, U. On new inequalities of Hermite-Hadamard-Fejér type for harmonically convex functions via fractional integrals. Springerplus 2016, 5, 635. [CrossRef]

12. Nwaeze, E.R.; Kermausuor, S. Caputo-Fabrizio fractional Hermite-Hadamard type and associated results for strongly convex functions. J. Anal. 2021, 29, 1351-1365. [CrossRef]

13. Set, E.; Sardari, M.; Özdemir, M.E.; Rooin, J. On generalizations of the Hadamard inequality for $(\alpha, m)$-convex functions. Kyungpook Math. J. 2012, 52, 307-317. [CrossRef]

14. Sarikaya, M.Z.; Set, E.; Yaldiz, H.; Başak, N. Hermite-Hadamard's inequalities for fractional integrals and related fractional inequalities. Math. Comput. Model. 2013, 57, 2403-2407. [CrossRef]

15. Sarikaya, M.Z.; Yildirim, H. On Hermite-Hadamard type inequalities for Riemann-Liouville fractional integrals. Miskolc Math Notes 2017, 17, 1049-1059. [CrossRef]

16. Sezer, S. The Hermite-Hadamard inequality for s-convex functions in the third sense. AIMS Math. 2021, 6, 7719-7732. [CrossRef]

17. Srivastava, H.M.; Kashuri, A.; Mohammed, M.O.; Baleanu, D.; Hamed, Y.S. Fractional integral inequalities for exponentially nonconvex functions and their applications. Fractal Fract. 2021, 5, 80. [CrossRef]

18. Valdes, J.E.N.; Rodriguez, J.M.; Sigarreta, J.M. New Hermite-Hadamard type inequalities involving non-conformable integral operators. Symmetry 2019, 11, 1108. [CrossRef]

19. Kunt, M.; İşcan, İ. New general integral inequalities for $(\alpha ; m)$-GA-convex functions via Hadamard fractional integrals. arXiv 2015, arXiv:1505.03318.

20. He, C.Y.; Wang, Y.; Xi, B.Y.; Qi, F. Hermite-Hadamard type inequalities for $(\alpha, m)$-HA and strongly $(\alpha, m)$-HA convex functions. J. Nonlinear Sci. Appl. 2017, 10, 205-214. [CrossRef]

21. Tunç, T.; Budak, H.; Usta, F.; Sarikaya, M.Z. On new generalized fractional integral operators and related fractional inequalities. Konuralp J. Math. 2020, 8, 268-278.

22. Kilbas, A.A.; Srivastava, H.M.; Trujillo, J.J. Theory and Applications of Fractional Differential Equations; North-Holland Mathematics Studies; Elsevier: New York, NY, USA; London, UK, 2006.

23. Jarad, F.; Ǔgurlu, E.; Abdeljawad, T.; Baleanu, D. On a new class of fractional operators. Adv. Difference Equ. 2017, $2017,247$. [CrossRef]

24. Samko, S.G.; Kilbas, A.A.; Marichev, O.I. Fractional Integrals and Derivatives-Theory and Applications; CRC Press: Boca Raton, FL, USA, 1993.

25. Miller, K.; Ross, B. An Introduction to the Fractional calculus and Fractional Differential Equations; John Wiley and Sons, Inc.: New York, NY, USA, 1993.

26. Mubeen, S.; Habibullah, G.M. k-fractional integrals and applications. Int. J. Contemp. Math. Sci. 2012, 7, 89-94.

27. Farid, G.; Rehman, A.U.; Tariq, B.; Waheed, A. On Hadamard type inequalities for $m$-convex functions via fractional integrals. J. Inequal. Spec. Funct. 2016, 7, 150-167.

28. Farid, G.; Rehman, A.U.; Tariq, B. On Hadamard-type inequalities for $m$-convex functions via Riemann-Liouville fractional integrals. Stud. Univ. Babeş-Bolyai Math. 2017, 62, 141-150. [CrossRef]

29. Özdemir, M.E. Some inequalities for the s-Godunova-Levin type functions. Math. Sci. 2015, 9, 27-32. [CrossRef]

30. Noor, M.A.; Noor, K.I.; Awan, M.U. Integral inequalities for harmonically s-Godunova-Levin functions. Facta Univ. Ser. Math. Inf. 2014, 29, 415-424.

31. Noor, M.A.; Noor, K.I.; Awan, M.U. Some inequalities for geometrically arithmetically h-convex functions. Creat. Math. Inform. 2014, 23, 91-98. [CrossRef]

32. İşan, İ. Ostrowski type inequalities for harmonically s-convex functions. Konuralp J. Math. 2015, 3, 63-74.

33. Jia, W.; Yussouf, M.; Farid, G.; Khan, K.A. Hadamard and Fejér-Hadamard inequalities for $(\alpha, h-m)$ - $p$-convex functions via Riemann-Liouville fractional integrals. Math. Probl. Eng. 2021, 2021, 12. [CrossRef]

34. Fang, Z.B.; Shi, R. On the $(p, h)$-convex function and some integral inequalities. J. Inequal. Appl. 2014, 2014, 16. [CrossRef]

35. Zhang, K.S.; Wan, J.P. p-convex functions and their properties. Pure Appl. Math. 2007, 23, 130-133.

36. Breckner, W.W. Stetigkeitsaussagen für eine klasse verallgemeinerter konvexer funktionen in topologischen linearen Räumen. Publ. Inst. Math. 1978, 23, 13-20.

37. Xi, B.Y.; Qi, F. Properties and inequalities for the $\left(h_{1}, h_{2}\right)$-and $\left(h_{1}, h_{2}, m\right)$-GA-convex functions. Cogent Math. 2016, 3, 1176620. [CrossRef]

38. Noor, M.A.; Noor, K.I.; Awan, M.U.; Costache, S. Some integral inequalities for harmonically h-convex functions. Politehn. Univ. Buchar. Sci. Bull. Ser. A Appl. Math. Phys. 2015, 77, 5-16.

39. Marian, D. On $h-E$-convexity. Autom. Comput. Appl. Math. 2010, 19, 305-312.

40. Youness, E.A. E-convex sets, E-convex functions, and E-convex programming. J. Optim. Theory Appl. 1999, 102, 439-450. [CrossRef] 